\title{
IDENTIFICATION OF THE TECHNICAL CONDITION OF ROLLER BEARINGS BY MEANS OF VIBRODIAGNOSTICS AND TRIBODIAGNOSTICS
}

\begin{abstract}
Summary
This paper focuses on determining the technical condition of a bearing box in the vehicle by means of vibrodiagnostics. In the next step, we determined the technical condition of roller bearings in the bearing box by measuring the vibration signal. One of the possibilities of determining the technical condition, including the location of accelerometers, the arrangement of relevant measured devices, and the analysis of frequency spectrum, is described in the paper. The aim of the experiment was to measure vibrations of the bearings placed in the vehicle bearing box and then to carry out the analysis of the measured data. The second part of the paper presents the analysis of samples of the plastic lubricant used for lubricating the tested roller bearings. For the analysis, we used an AES Spectroil Q100 device which works on the principle of atomic spectral analysis.
\end{abstract}

Keywords: vibrodiagnostics, tribodiagnostics, roller bearing, spectral analysis, envelope detection, Fourier transform, frequency of damaged bearings

\section{Introduction}

A bearing is one of the most important and widely used machine spare parts. During the continuous monitoring of its technical condition it is possible to make a plan of predictive maintenance which can prevent unexpected breakdowns and failures.

Although there are a number of many more diagnostic ways to determine a technical condition, when it comes to determining the condition of a bearing, a vibrodiagnostic signal seems to be the most suitable. Bearing vibration is always connected with the dynamic loading of specific roller bearing parts, e.g. an outer or an inner ring, roller elements, or a snap ring. The technical condition is also affected by non-alignment, unbalance, wear, etc. The observation and the analysis of vibrations enable us not only to verify the dynamic loading of roller bearing parts, but also locate the place where the extreme vibrations occur (it means the malfunctions of bearing parts) and therefore eliminate the cause of these vibrations.

At present, vibrodiagnostics is developing rapidly, applying very up-to-date diagnostic ways. When it is used for detecting the bearing technical condition, it often uses excellent software support. The largest step ahead in this area is the utilization of information technologies which allows us to perform measurements and evaluation in real time. 
The level of mechanical oscillation of a device can be described by the bathtub curve. The bathtub curve describes operating conditions in which a device can be during its life. These conditions correlate with vibrations which cause oscillations of different amplitudes, velocity, and acceleration.

Vibrodiagnostics is one of the most important methods used for identifying a technical state. With the use of vibration diagnostics we are able to detect an incipient failure, to locate the place of an incipient failure and to predict the length of time during which a device is going to work before a failure occurs or a preventive action is performed. The proper application of vibrodiagnostics can prevent undesirable damage to machines, thereby preventing costs associated with repair and wasting money when a device is out of service.

Experimental vibrodiagnostic measurements are performed while obtaining primary signals. Primary results of measurements are systematized and analysed. Operating characteristics of rotary systems with bearings of sliding friction and those of roll are compared. Generalization of research results is done and conclusions are formulated, [2],[13],[12].

Bartelmus and Zimroz introduced a new diagnostic feature, which can be used for monitoring the condition of planetary gearboxes in time-variable operating conditions [1]. This diagnostic feature is the sum of 10 amplitudes of spectral components from the spectrum of the gearbox vibration signal. Unfortunately, this vibration diagnostic feature is useful only in a limited range of operating conditions. Bartelmus and Zimroz stated that the recognition of different technical conditions is very difficult or impossible in the cases of no load or small load [1].

Two types of excitations can produce noise in rotating machinery, i.e. excitations due to primary sources and excitations due to manufacturing defects. It is difficult to act on the primary sources or functional loads as they are specific to a machine. These defects are inherent in both the manufacturing quality and the configuration of the machine. The defects caused by the rotating parts of the machine (rotors, disks, bearings, gears, etc.) create concentrated or distributed loads that produce vibrations and noise. The vibrations depend on the machine itself through the transfer function while the noise is a function of the machine environment [7].

Gearbox noise often contains a wide range of frequencies within the audible range. Advanced signal processing techniques have been applied to an experimental vibro-acoustic analysis of helicopter gearboxes to identify the vibrations caused by gears, shafts, and bearings [7]. On the other hand, computational vibro-acoustic analysis of geared systems can rarely be found in the literature [1]. Structure-borne gearbox noise originating from gear vibration is often estimated empirically [9]. These acoustic estimates are specific to the experimental gearbox that was analysed and may not be suitable for other gearboxes because the generated noise depends strongly on the shape of the housing [6]. Advanced computational models suitable for the development of a modern powertrain in the field of noise and vibration are presented in [15]. The aim is to decide how detailed the model should be to correctly describe the vibrational and acoustic performance of the powertrain.

A lot of companies spend large amounts of money on the preventive maintenance of machines and technical equipment despite the fact that there is no need to take a preventive action at that particular moment. This leads to massive financial losses, mainly during the time the machine is out of service. Therefore, it is desirable to perform preventive maintenance at the time when it is really necessary (Fig. 1). A decision on taking a preventive action or replacing a part which is based on the real technical condition is proactive maintenance. This is made possible by using a mathematical model describing the technical condition of a machine during its life. The aim of our paper is to describe the technical condition of the vehicle bearing box by means of vibrodiagnostics [3]. 


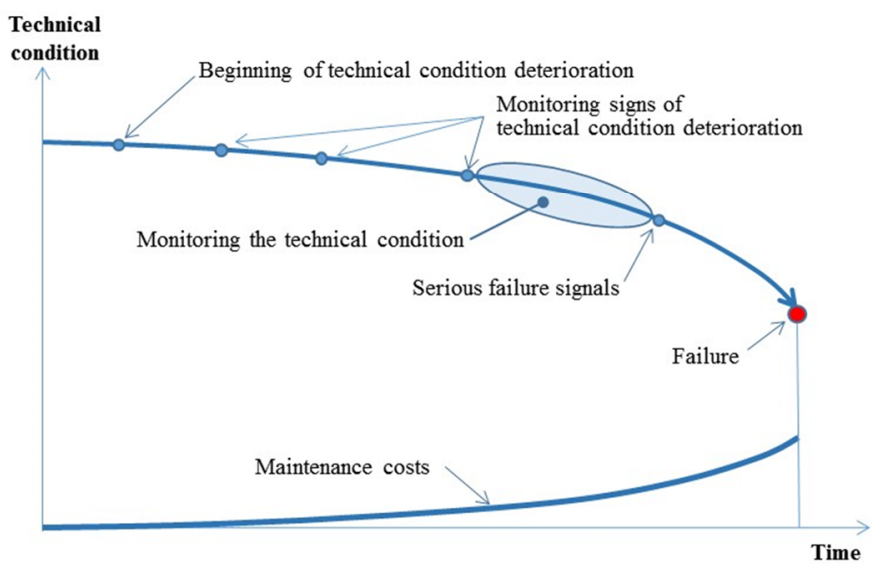

Fig. 1 Diagram of time-dependent predictive maintenance [4]

\section{Possibilities of determining the technical condition of rolling bearings}

\subsection{Main characteristics of vibrations}

Vibrations occur as a result of rotating or straight-line moving bodies. The course of vibrations is influenced mainly by the technical condition of individual machine components such as shafts, gear boxes, crank mechanisms, cam mechanisms, antifriction bearings, and also by the imbalance of rotating parts, backlash in friction bearings, wear, material fatigue, cracking phenomenon, corrosion, and other factors affecting the smooth running of a machine. The vibration itself is defined then as a dynamic phenomenon when particles or solid bodies move around a zero equilibrium position. Vibrations are given by a combination of six movements, namely by a shift in an orthogonal coordinate system $\mathrm{x}, \mathrm{y}, \mathrm{z}$ and by the rotation about these axes. We can describe them by the amplitude and phase in a certain period of time. Depending on the time variations of values, vibrations are of a periodical, nonperiodical, or random character. As for periodical vibrations, the course of vibrodiagnostic values keeps repeating over time. Harmonic vibration, which has a sinusoidal waveform, is based on these vibrations. For harmonic vibrations we need to set only one determining value and the others can be calculated.

The basic way of describing oscillations is to determine their displacement $x$, velocity $v$, acceleration $a$, maximum value $A_{\max }$, the root of mean square $R M S$, and the average value $A_{\text {ave }}$ (Fig. 2).

The measurement of displacement $x$ is convenient for low-frequency phenomena, such as backlashes. The displacement $x$ can be calculated in the following way:

$$
\begin{aligned}
& x=X_{\text {max }} \sin \omega t \\
& \omega=2 \pi f
\end{aligned}
$$

where $X_{\max }$ is the maximum displacement, $\omega$ is the angular frequency, $f$ is frequency (oscillation), $t$ is time.

Velocity can be expressed as the characteristics of motion which informs us about the way the position of a body (particle) changes in time. Velocity is a vector quantity because it defines both the magnitude and direction of the change. Velocity can be determined as the time derivation of trajectory (displacement) using the equation below:

$$
v=\frac{\mathrm{d} x}{\mathrm{~d} t}=X_{\max } \omega \cos \omega t
$$


Acceleration can be expressed as the characteristics of motion which shows the way the velocity of a body (particle) changes in time. Acceleration is a vector quantity since it gives both the magnitude and direction of the change. It is possible to calculate the instantaneous acceleration and the average acceleration. Acceleration may also be determined as the time derivation of velocity using the formula below [5]:

$$
a=\frac{\mathrm{d} v}{\mathrm{~d} t}=-X_{\max } \omega^{2} \sin \omega t=X_{\max } \omega^{2} \sin (\omega t+\pi)
$$

If acceleration is related to a change in velocity in terms of a decreasing speed, it is called deceleration and has a minus sign. The mean absolute value $A_{\text {ave }}$ can be expressed as follows:

$$
A_{\text {ave }}=\frac{1}{T} \int_{0}^{T}|a| \mathrm{d} t
$$

where $T$ is the period expressed by the formula $T=\frac{1}{f}$.

The International Standards Organization (ISO), which establishes internationally acceptable units for the measurement of machinery vibration, suggested the root mean square (RMS) as the standard unit of measurement. This was decided in an attempt to establish criteria that would determine an effective value for the varying function of acceleration. Acceleration tends to provide the energy content in the vibration signal, whereas the acceleration peak is correlated better with the intensity of vibration.

The root of mean square can be calculated by the equation below [12]:

$$
R M S=\sqrt{\frac{1}{T} \int_{0}^{T} a^{2} \mathrm{~d} t}
$$

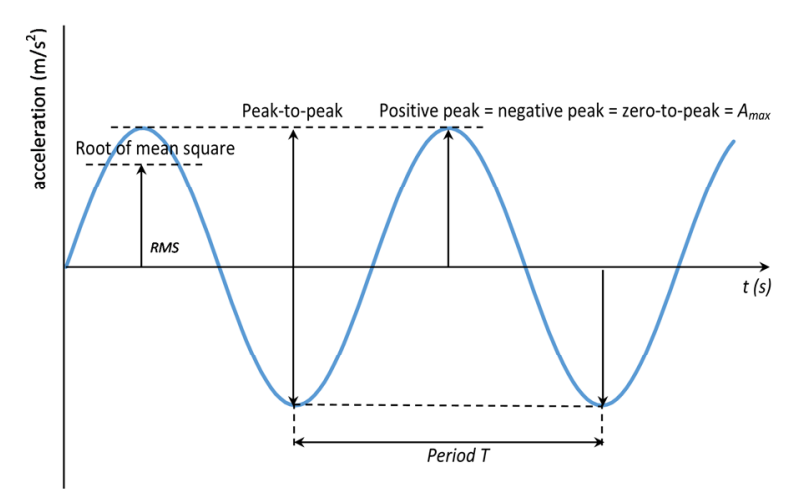

Fig. 2 Course of the signal and calculated $R M S$ [12]

In order to interpret the measured values correctly, it is advisable to transform the oscillation time course into a frequency domain, i.e. vibrations are to be replaced by a sequence of their harmonic components. It can be said that the time signal contains the information about when a certain event occurred, but the frequency spectrum contains the information about how often the same event occurs in the observed signal. The procedure during which complex signals are subdivided into their frequency components is called a frequency analysis; it uses either selective band-pass filters or more often the fast Fourier transform (FFT). Along with the FFT, also a wavelet, a cosine or the Walsh-Hadamard transform can be used for expressing a signal by orthogonal basis functions. In this study, we have applied the fast Fourier transform. 


\subsection{The use of tribodiagnostics}

Tribology is the science which applies the knowledge based on research into friction, wear and lubrication to decrease friction coefficient, or to optimize the course of friction, thereby reducing the wear of interacting surfaces of elements in relative motion. Tribodiagnostics is a non-destructive method which focuses on the lubricant as a source of information about the condition of and changes in mechanical systems in which it is applied. Tribodiagnostics deals with two basic areas:

1. Determining the condition, prolonging the usability, and predicting the degradation of lubricants.

2. Determining the regime, location, and trend of the mechanical system wear by evaluating quantitatively and qualitatively the amount of abrasive particles in the lubricant.

During the experiment we observed the operating degradation of plastic lubricant during the life of an anti-friction bearing. This was achieved by applying regular methods (standardized) or special tribodiagnostic methods. Generally speaking, the determination of wear of mechanical systems lubricated by plastic lubricants is based on the assumption that after some operating time each plastic lubricant contains a certain percentage of adulterants. The majority of particles dispersed in the plastic lubricant are metal particles generated by abrasive wear. When quantifying, we use a suitable method which enables us to observe indirectly mechanical changes in the system. The number of metal particles their growth rate, shape, morphology, size, and composition help us to draw particular conclusions. If the growth and other parameters match the nominal values determined for a given mechanical system, it might be concluded that there is a regular course of wear without an increased risk of a system failure. The excessive or sudden growth of the number or the size of metal particles indicates an unusual situation. The size, the shape, the rate of growth and other parameters help us to identify the failure severity and the necessity to perform corrective actions. If we know what material the parts of the system lubricated by a plastic lubricant are made of, we are able to determine and locate a frictional pair by the type of metal abrasion. The frictional pair shows a sharp increase in wear. Regular monitoring of the content of the so-called signal metals and particles enables us to predict the occurrence of failures. In order to determine the content of metals and other elements, different methods of instrumental analysis are used. The most widely used methods are spectroscopy and polarography. The course and the extent of wear of parts lubricated by a relevant lubricant are observed by applying other methods suitable for the description of the morphology and the division of particles formed by metal abrasion, of fibres from filtering materials, of contaminants from the environment, etc. The method used in our study is ferrography which applies an image analysis.

\section{Characteristics of vibration sensors}

Vibration sensors are used for measuring physical values (displacement, velocity and acceleration) and for transforming them into electric signals which are later used to work with. There is a great variety of different vibration sensors which are used for achieving results of highest possible level of accuracy, such as acceleration, velocity, and displacement sensors. The principle of operation of a vibration sensor is the motion of seismic matter of mass $\mathrm{m}$ towards an object of mass $\mathrm{M}$ whose vibrations are measured. For calculation, the following formula is used

$$
m a_{h}+b v+k y=-m a_{0}
$$

where $y$ - displacement, $v$ - velocity, $a_{h}$ - acceleration of seismic matter motion, $a_{0}$ - object acceleration, $m$ - seismic matter mass, $k$ - spring stiffness, $b$ - damping coefficient [5]. 
For the measurement, we used full equipment of the Dewetron Company including the evaluation software DEWESOFT. We used a DEWE-50-USB2-8 device which is an 8channel measuring system where eight slots might be connected for modules DAQ and PAD.

For the same purpose, we used two triaxial acceleration sensors which are at present basic vibration sensors used for performing measurements mainly on the fixed parts of rotating machines like in this case. We used KS943B.100 sensors which are placed in a duralumin case. The sensors have the measuring range $\pm 60 \mathrm{~g}$, measuring sensitivity of 100 $\mathrm{mV} / \mathrm{g}$, and frequency range $0.5 \mathrm{~Hz}-22 \mathrm{kHz}$ (Fig. 3).

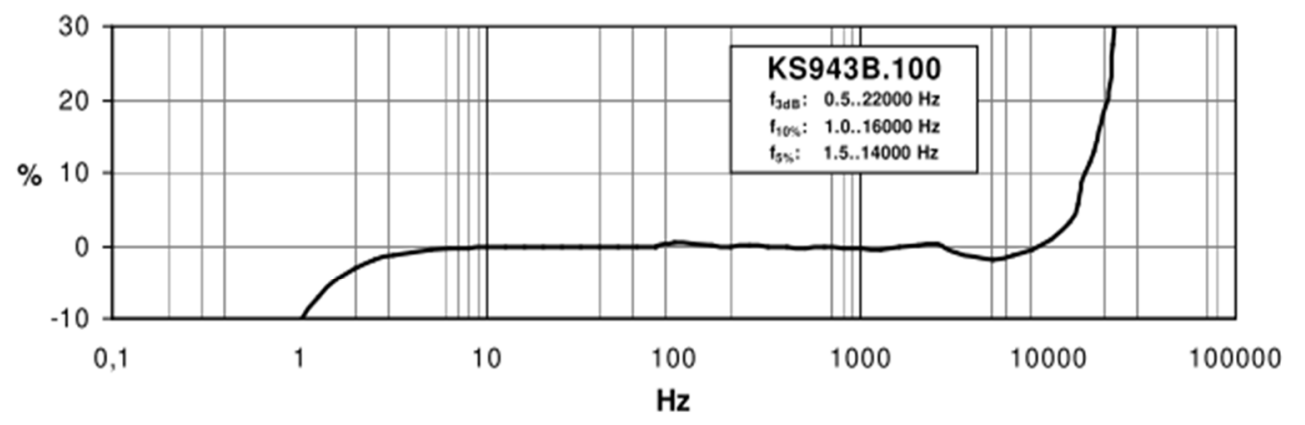

Fig. 3 Calibration curve of the KS943B.100 accelerometer [14]

If the measurement is to be accurate, it is necessary to select the right place for measuring and the right way of attaching sensors. Sensors are placed on clean surfaces as close to the place where vibrations occur as possible. If the sensors are attached inappropriately, the measured data may be completely unreliable, or the applicable frequency range of a sensor can be significantly limited.

\section{Description of the approach to determining the technical condition of a measured bearing}

Most of the damages to roller bearings occur because of wear. As a result, fatigue of single components occurs, which leads to the chipping of the surface layer material (pitting); this, in turn, results in increased wear and clearance, corroding process, deepening of the grooves, and failures of snap rings. Every time one of the rolling elements is damaged in any way, it starts to pulse, thereby stimulating natural frequencies in bearing rings. The speed of repetitive pulses causes a failure frequency which enables us to locate the damage to the roller bearing. The calculations of the failure frequencies are in Eq. (8) - (11). For this, it is necessary to know the type of a bearing and its number. In the bearing box of the vehicle, the roller bearing is designated as $31308 \mathrm{~A}$.

For the kinematic frequencies of impulses, provided the motion is all rolling, the formulae [8] apply:

a) BPFO - Ball Pass Frequency - Outer Race (outer ring defect)

$$
f=\frac{n}{2} f_{r}\left(1-\frac{B D}{P D} \cos \beta\right)
$$

b) BPFI - Ball Passing Frequency Inner Race (inner ring defect)

$$
f=\frac{n}{2} f_{r}\left(1+\frac{B D}{P D} \cos \beta\right)
$$


c) BSF - Ball Spin Frequency (bearing defect - of a bearing ball or a bearing roller)

$$
f=\frac{P D}{2 B D} f_{r}\left(1-\left(\frac{B D}{P D} \cos \beta\right)^{2}\right)
$$

d) FTF - Fundamental Train Frequency (snap ring defect)

$$
f=\frac{1}{2} f_{r}\left(1-\frac{B D}{P D} \cos \beta\right)
$$

where $n$ - the number of bearing balls or rollers, $f_{r}$ - the frequency given by the relative revolution (speed) of the inner and the outer ring, $B P$ - the diameter of a bearing ball or roller, $P D$ - the pitch diameter, $\beta$ - the contact angle [11].

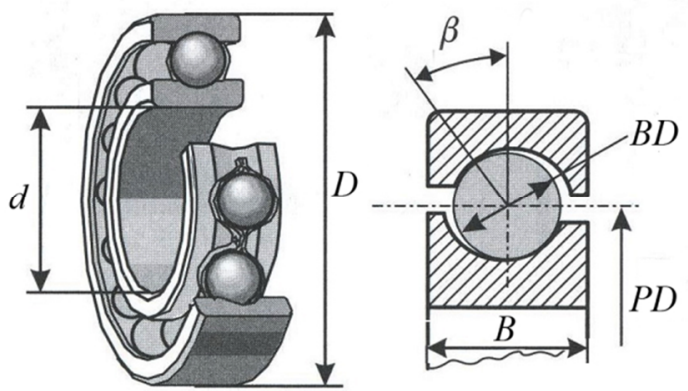

\begin{tabular}{|l|c|c|}
\hline Outer diameter & $D$ & $90 \mathrm{~mm}$ \\
\hline Inner diameter & $d$ & $40 \mathrm{~mm}$ \\
\hline Width & $B$ & $23 \mathrm{~mm}$ \\
\hline Pitch circle & $P D-d_{l}$ & $63.3 \mathrm{~mm}$ \\
\hline Contact angle & $\beta$ & $24.374^{0}$ \\
\hline Number of rolling elements & $N$ & 16 \\
\hline Diameter of a rolling element & $B D$ & 11.74 \\
\hline
\end{tabular}

Fig. 4 Cross-section of the roller bearing 31308 with relevant parameters [10]

Damage to bearings is generally divided into four stages, $1-4$, see Fig. 5. Also, the spectrum of deteriorated bearings is divided into four zones (A, B, C, and D) which are as follows:

Zone A expresses the input RPM and subsequent harmonic components.

Zone B expresses the failure frequency of a roller bearing (about 0.1 up to $3.5 \mathrm{kHz}$ ).

Zone $\mathrm{C}$ expresses the vibration frequencies of a roller bearing (about 3.5 up to $20 \mathrm{kHz}$ ).

Zone D expresses a high frequency zone (HFD) where the oscillation frequency is higher than $20 \mathrm{kHz}$.
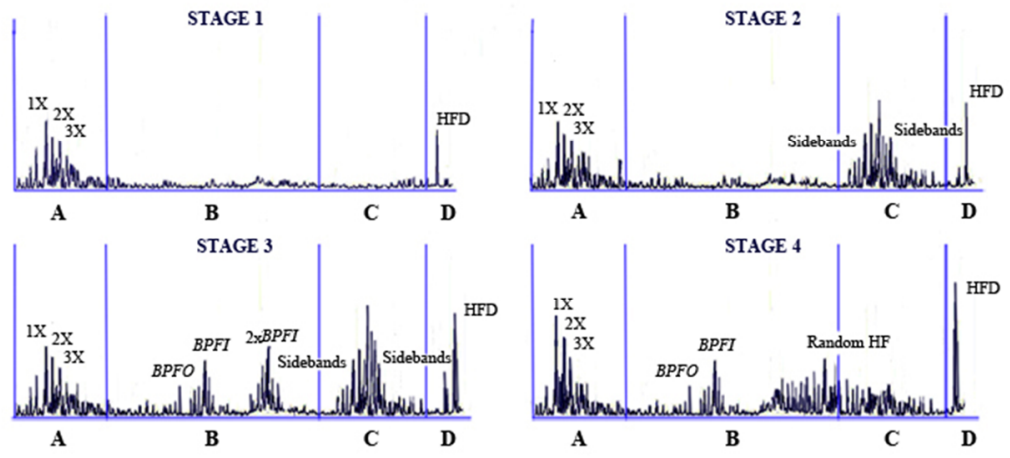

Fig. 5 Stages and zones of bearing damage [5]

In the initial stage, the damage is extremely small and the deterioration rate is constant. In this stage, there are no frequencies in zones B and C.

During the second stage, small bearing defects start to occur and the deterioration rate increases slightly. In the roller bearing, the frequencies of bearing components themselves start occurring and range mostly between $0.1 \mathrm{kHz}$ up to $20 \mathrm{kHz}$. At the end of the second 
stage, side bands appear above and under the resonance peak. The HFD might double as compared with the value of amplitudes in the initial stage.

In the third stage, the failure bearing frequencies of defects and their harmonic elements start occurring. As the wear of roller bearings increases, the harmonic elements of failure bearing frequencies begin to go up and the number of side bands goes up as well. The HFD might almost double as compared with the value of amplitudes in the second stage. In this stage, we try to prevent the failure and we replace the roller bearing. Otherwise, the bearing would fail causing damage to other components as a result of the subsequent failure of the bearing box.

In the final stage, the discreet bearing frequencies of failures and bearing frequencies themselves are replaced by the random broadband high frequency oscillation.

In practice, there are some other methods used for determining the technical condition of roller bearings, e.g. the Shock Pulse Method (SPM) of the SPM company, the Bearing Condition Unit (BCU) method of the Schenck company, the Spike Energy (SE) method, the method of using the $K_{(t)}$ parameter, the envelope detection method, etc. [5].

\section{Description of the experiment}

The measurement was performed on a testing stand shown in Fig. 6. The main parts of the stand are: a frame for anchoring measured equipment (1), an asynchronous motor with engine speed control in $10-3000 \mathrm{RPM}(2)$, a propeller shaft with a flange (3), a frequency converter MOVITRAC MCLTE B0040-5A3-4-00 (4), measuring equipment DEWE-50USB2-8 (5), a notebook with software DEWESOFT X2 (6), two tri-axial acceleration sensors KS943B.100 (7), and measured equipment (8). We used a vehicle bearing box.

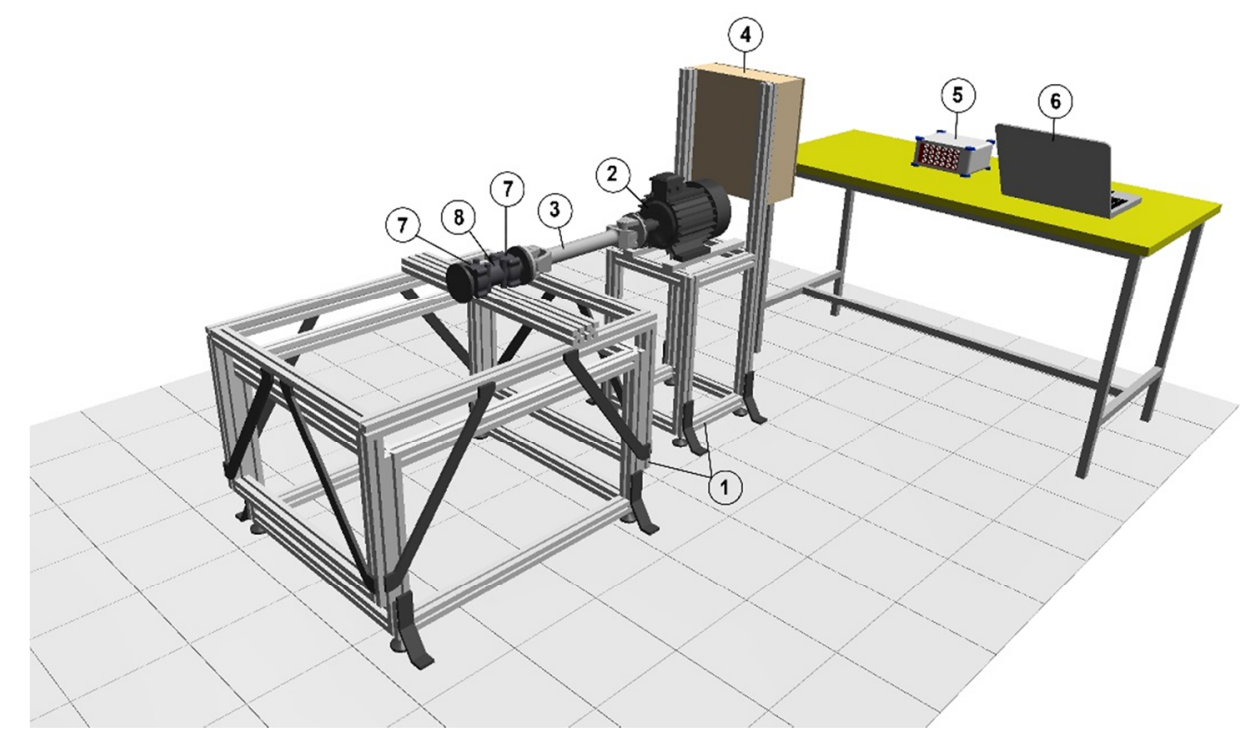

Fig. 6 Testing stand for vibration measurements

For the measurement itself, we used the DEWE-50-USB2-8 device from the DEWETRON firm. This is an 8-channel device intended for measuring vibrations and temperatures of equipment. The vibration signal was measured by two KS943B.100 tri-axial accelerometers by which it was possible to measure the vibrations of two bearings at the same time. These accelerometers were mounted on the bearing box by means of a screw bolt, see Fig. 3. The surface under the accelerometer should have been cleaned and smoothed so that the acceleration meter could be in full contact with the measured device. The drilled hole for the screw bolt should be perpendicular to the measured surface and sufficiently deep, see Fig. 6. 


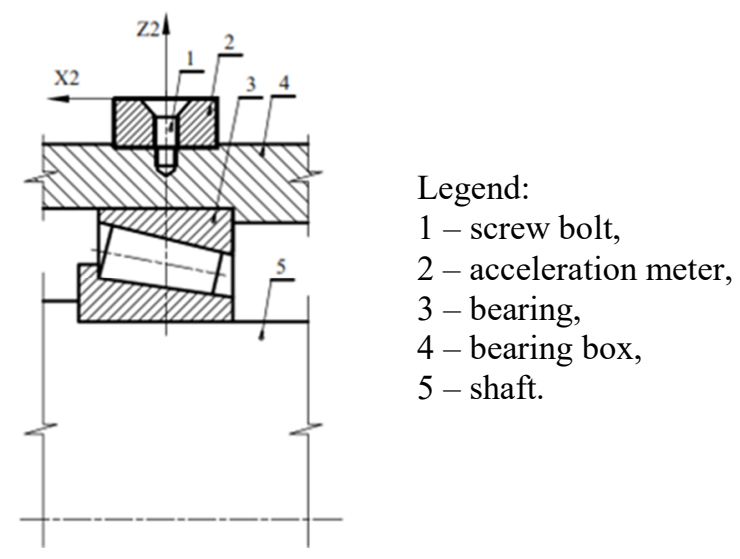

Fig. 7 Mounting of the accelerometer during measurement

Fig. 8 shows a simplified diagram of the measuring device.

\begin{tabular}{|c|c|c|c|c|}
\hline \multirow{2}{*}{$\begin{array}{l}\text { Acceleration } \\
\text { sensor } \\
\text { KS943B.100 }\end{array}$} & $\begin{array}{l}\text { Cable with } \\
\text { connectors }\end{array}$ & \multirow{2}{*}{$\begin{array}{c}\text { Measuring device } \\
\text { DEWE-50-USB2-8 } \\
\text { including amlifier } \\
\text { DAQP-ACC-A }\end{array}$} & \multirow[t]{2}{*}{ Cabel USB } & \multirow{2}{*}{$\begin{array}{l}\text { Computer with } \\
\text { DEWEsoft }\end{array}$} \\
\hline & & & & \\
\hline
\end{tabular}

Fig. 8 Diagram showing the measuring elements of the experiment

In order to evaluate the measurement of vibration signals we used the envelope detection analysis. This method makes it possible to detect not only the damage to the bearing, but also, using the fast Fourier transform, to determine which part of the roller bearing is actually damaged. That means that we may find out whether the failure occurred in a roller element, the inner or the outer ring, or the cage. This is based on the assumption that each part listed above is of a different relative speed to the shaft and of a different frequency at which the failures occur. Therefore, impulses which occur when the rolling element track is damaged should be measured. The signal in the time zone is filtered by a band-elimination filter covering the frequency range in which we found an increase in the amplitudes of the monitored spectrum components. The signal in the output of the band-elimination filter contains only the elements with high frequencies which definitely include vibrations, induced by impulses due to a failure. Other "masking" elements are effectively suppressed. The output signal of the band-elimination filter is further processed using a low-pass filter rectifier with a limiting frequency which corresponds approximately to the half of the width of the filter pass band. The most important, however, is the fact that in the worked signal, the initial pulserepetition frequency is safely renewed.

\section{Analysis of measurement results}

The bearing box was in operation for less than 3200 hours during which period it would have covered $122500 \mathrm{~km}$. These values were calculated on the basis of the known speed ratio in the gearbox and the auxiliary gearbox. During the experiment, the bearing box worked in different rotation modes during which we simulated the vehicle operating conditions. The individual measurements were based on the relevant methodology dealing with measurements. During the measurement, we always found the vibration values at 1000, 1200, 1400, 1600, 2200, 2600, and 3000 RPM of the input shaft. The experiment was performed only for the vibrations at 1200 RPM and 2220 RPM of the input shaft. 
6.1 Influence of the roller bearing life on the vibration signal

When evaluating the dependence of the roller bearing life (mileage) on the acceleration amplitude at a defined input constant of 1200 RPM, only five measurement results were chosen, namely those at 0, 30000, 60000, 90000 and 122500 kilometres. During the experiment the roller bearing was not adjusted, only lubricant was changed at regular intervals. When evaluating the experiment, we used the value on "Z2" axis which is the vertical line, see Fig. 9.

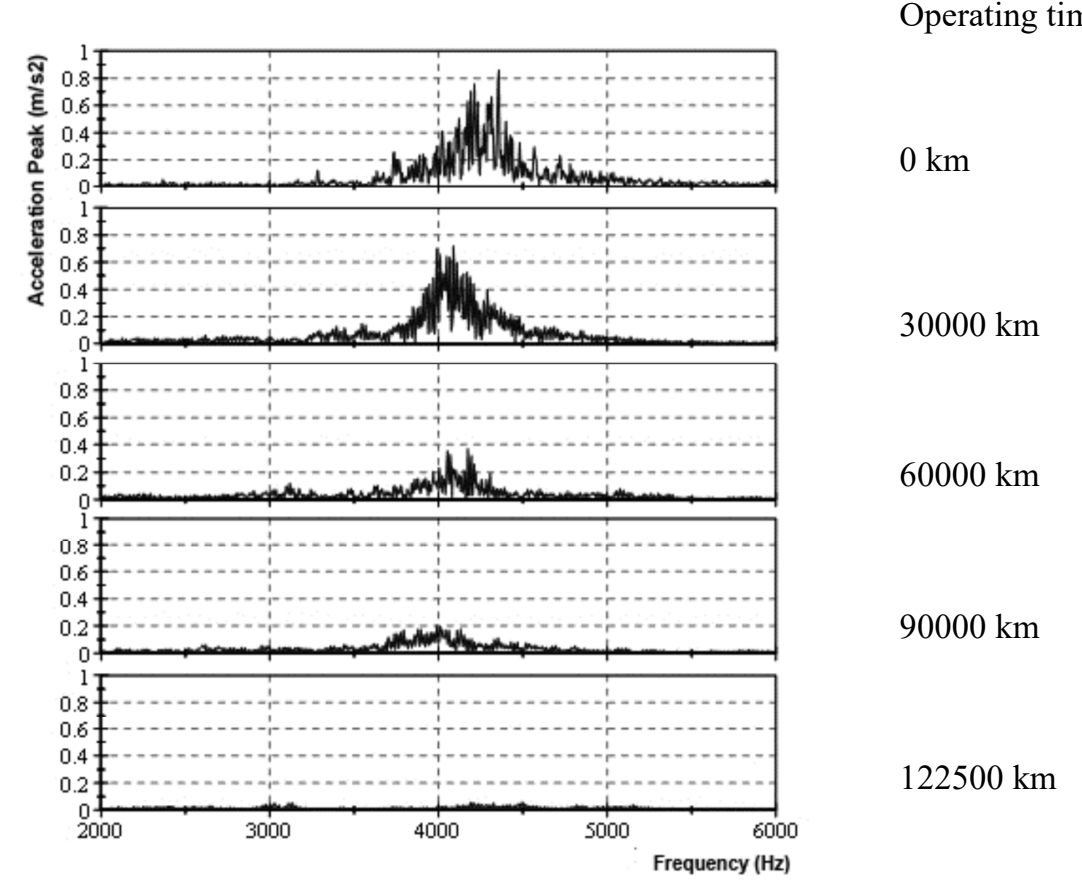

Fig. 9 The frequency spectrum recorded at different operating times

For the analysis of the experiment, we selected the area of $3.5 \mathrm{kHz}$ to $5 \mathrm{kHz}$ in which the bearing frequency is manifested. Based on the results (see Fig. 9), we can state that the longer the life (mileage) of the roller bearing in the bearing box of the unloaded vehicle is, the lower is the amplitude in the frequency analysis. This specific situation may be caused by the unloaded roller bearing which resulted in an increase in the bearing clearance in proportion to the operation, as it has been mentioned. From the above measurement conditions of the experiment one can see that the vibration of the rolling bearing in this area, depending on the mileage, is nonstandard. Therefore, it is necessary to adjust the bearing operation periodically.

At the end of the experiment, we examined the rolling elements under a microscope and discovered that they showed signs of wear. It is obvious that the operation resulted in the wear which is still far from the failure limit (Fig. 10). The rolling bearing was damaged only locally, which does not have a negative impact on its further operation. We came to a conclusion that after the $122500 \mathrm{~km}$ mileage, the rolling bearing is still able to fulfil its function. The measurement results presented in our paper also support our conclusion. 

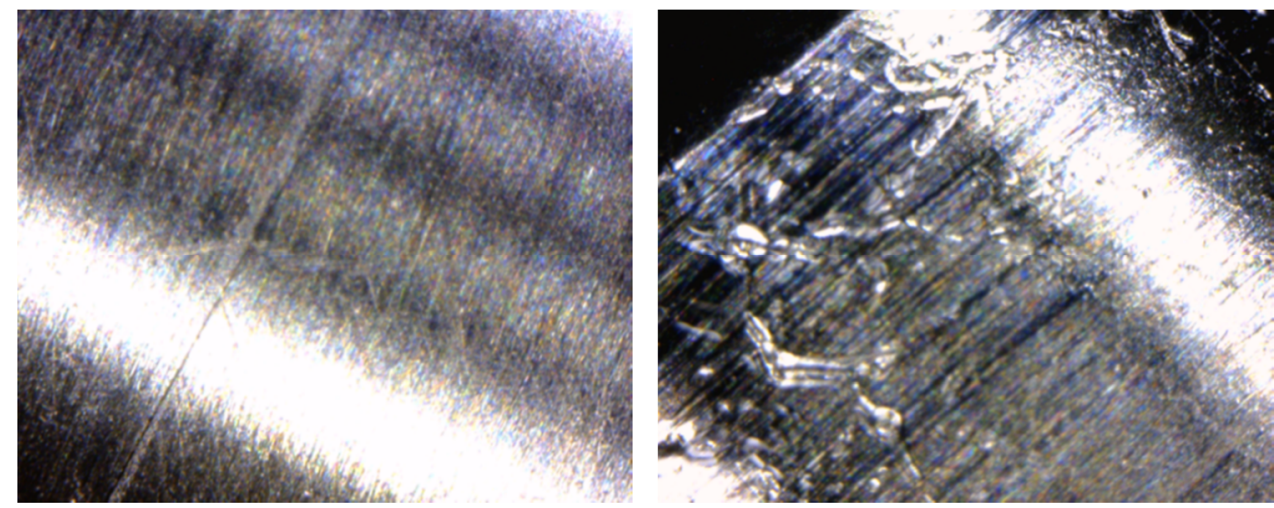

Fig. 10 Damage to the edges of the rolling element (left), and imprinted on the circumference of strip element (right), magnified 200x.

At this moment, we finished the experiment since the service life of military vehicles with this particular bearing box is only $50000 \mathrm{~km}$.

\subsection{Influence of the damaged outer ring on the vibration signal of a roller bearing}

In order to verify the influence of the damaged outer ring on the vibration signal of the tested roller bearing, the outer ring was artificially damaged. We cut a groove parallel to the axis of rotation and perpendicular to the track of rolling elements, see Fig. 11. For the experiment analysis, we selected the measurement at $1200 \mathrm{RPM}$ of the shaft and the second bearing on the "Z2" axis. The failure occurred in a similar way on the "X2" and "Y2" axes.

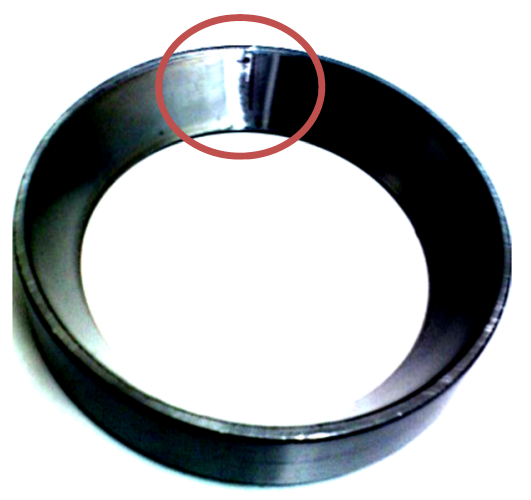

Fig. 11 A groove created on the outer ring

Table 1 The values calculated for bearing fault frequencies labelled FAG 31308A

\begin{tabular}{|l|c|c|c|c|}
\hline $\begin{array}{c}\text { Designations of fault } \\
\text { frequency }\end{array}$ & $\begin{array}{c}f_{r} \text { frequency } \\
20[\mathrm{~Hz}]\end{array}$ & $\begin{array}{c}f_{r} \text { frequency } \\
26.66[\mathrm{~Hz}]\end{array}$ & $\begin{array}{c}f_{r} \text { frequency } \\
36.66[\mathrm{~Hz}]\end{array}$ & $\begin{array}{c}f_{r} \text { frequency } \\
50[\mathrm{~Hz}]\end{array}$ \\
\hline Snap ring defect $-F T F$ & 8.42 & 11.22 & 15.43 & 21.05 \\
\hline Bearing defect $-B P F$ & 107.22 & 143.72 & 194.63 & 269.55 \\
\hline Outer race $-B P F O$ & 136.64 & 179.48 & 246.80 & 336.6 \\
\hline Inner race $-B P F I$ & 185.36 & 247.08 & 339.76 & 463.4 \\
\hline
\end{tabular}

Table 1 shows the calculation results of the frequencies of typical bearing failures for the shaft revolving at $1200 \mathrm{RPM}$. The value of $B P F O$ corresponds to the result of the frequency analysis, which is put in the upper right hand corner in Fig. 12. Small errors may be caused by the inaccurate measurement of bearing shaft revolutions. Fig. 12 was created after having used the envelope detection which, on the basis of a band-elimination filter, enables us to filter the lower and upper undesirable vibrations which may have a negative impact on the envelope detection analysis. 


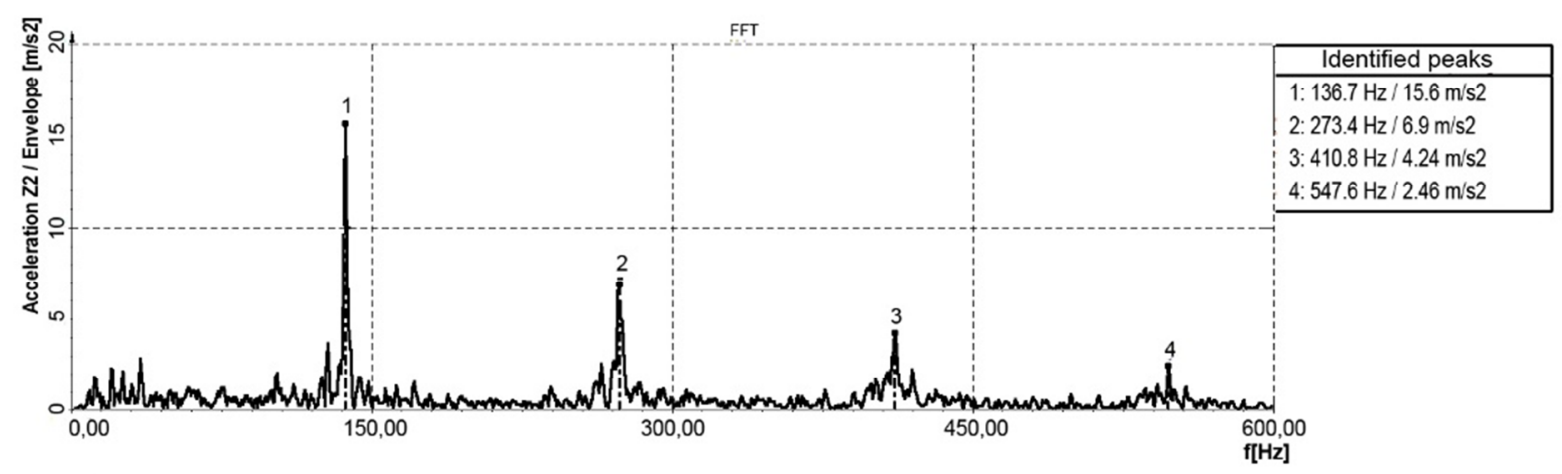

Fig. 12 The frequency spectrum of the damaged outer ring obtained by means of envelope detection

The experiment presented above confirmed the calculated value of outer race according to Eq. (8). The calculated $B P F O$ value is shown in Tab. 1. The realistic measured values correspond with identified peaks in Fig. 12. We have come to the conclusion that the envelope detection used for determining the frequency spectrum of roller bearing damage is the right method. In Fig. 12 there are the harmonic series (points 2, 3, and 4) and also the frequency spectrum of the outer ring damage (point 1).

In this experiment we also counted the RMS values in all three axes $(\mathrm{x}, \mathrm{y}, \mathrm{z})$ for both roller bearings. These values were summarized and expressed depending on the mileage. Fig. 13 shows the bearing further away from the electromotor (Fig. 6) marked with the number 2 where we measured the vibration signal at 1200 and 2200 RPM at regular intervals and calculated the RMS values. After the bearing had reached the mileage of $122500 \mathrm{~km}$, the bearing was artificially damaged, see Fig. 11, which resulted in a significant increase in the RMS value.

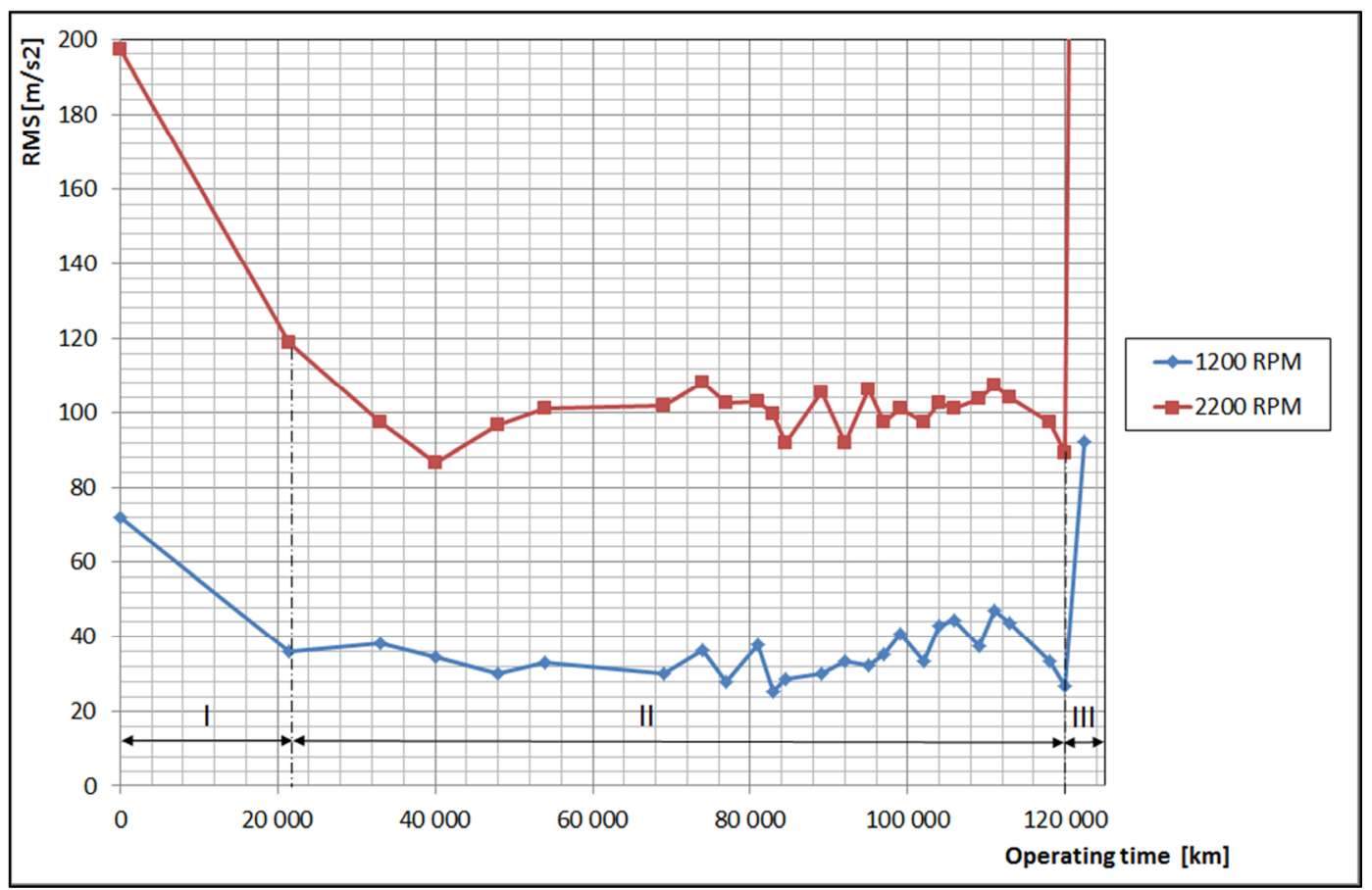

Fig. 13 Damaged bearing 2 - expressing the dependence of $R M S$ on mileage

Figure 13 shows that this limiting value will differ depending on RPM. Based on the measurements, in Fig. 14, the RMS limiting value of $85 \mathrm{~ms}^{-2}$ was determined for both bearings at 1200 RPM. 
Figure 14 shows the values of $R M S$ for the bearings 1 and 2 during the mileage of $122500 \mathrm{~km}$ at $1200 \mathrm{RPM}$. These graphs show that at first there is a period of running-in and positioning the bearings against each other with the $R M S$ value going down. During the other period characterized as operating time, the $R M S$ becomes more or less constant. In the third period of the bearing life, when the bearing becomes considerably worn, an artificial damage was done to the bearing, as it has been mentioned before. This led to a significant increase in $R M S$, which had been expected.

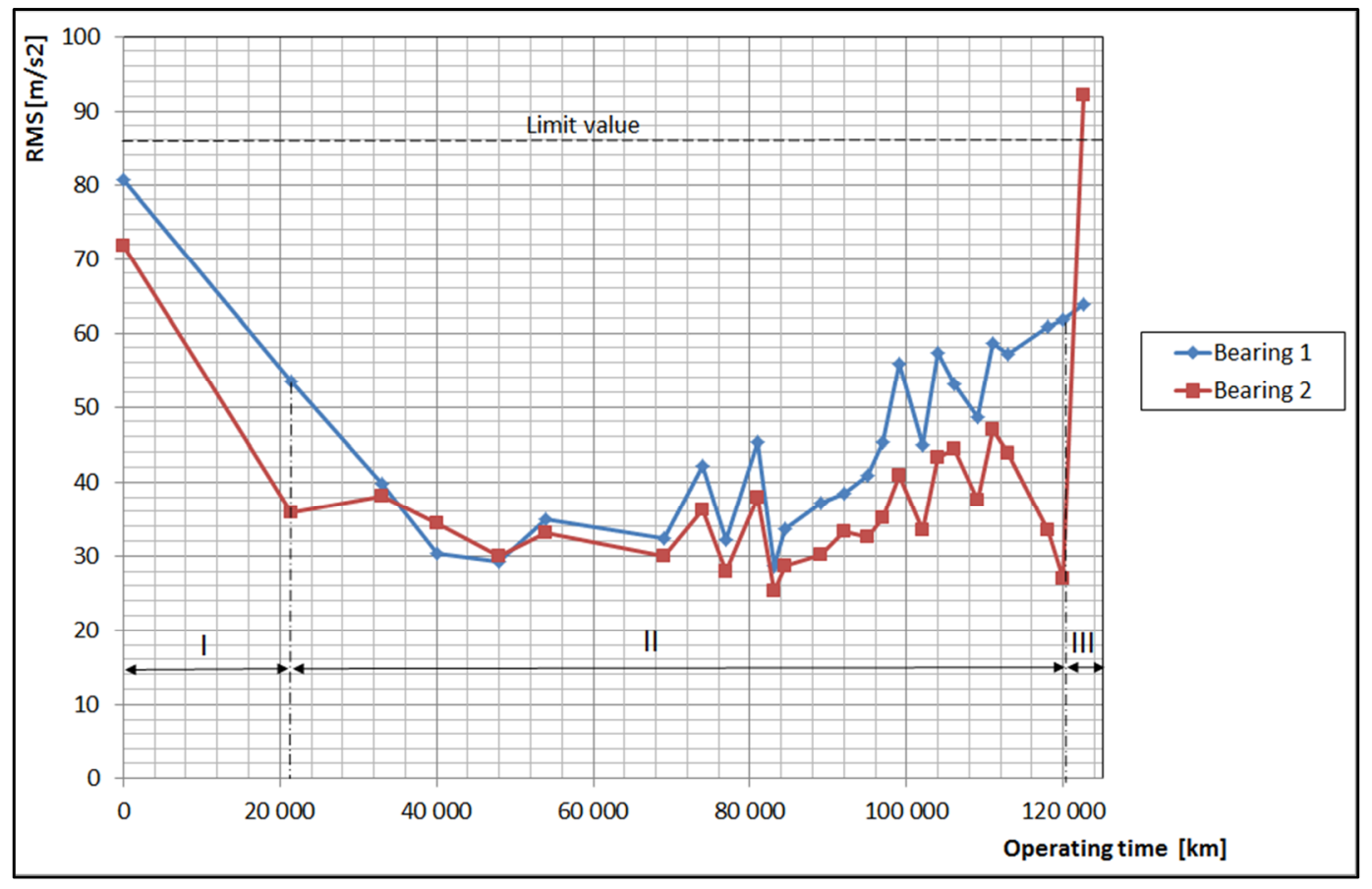

Fig. 14 Dependence of $R M S$ on the mileage at 1200 RPM for the bearings 1 and 2

\subsection{Influence of rolling bearing life on the lubricant}

Atomic emission spectrometry was used in our experiment to determine the quantity of certain elements in samples of lubricant. In this method, a sample of lubricant was exposed to light emitted from a spark or an arc, was turned into a gas state and atomized. By absorbing an amount of energy or by colliding with one another, the electrons of single atoms change from a basic state to an excited state. During the reverse transition to the basic state, the atoms radiate energy. This energy is the sum of respective atomic energy levels which is emitted in the form of light. The value of the light wavelength is characteristic of each element.

When determining the technical condition of the tested rolling bearing of the vehicle rolling box we used the MULTIFAK EP2 plastic lubricant. The bearing box was put through a demanding test on a test stand. The experiment took 3300 operating hours and this trial period equals to $125000 \mathrm{~km}$ covered by a vehicle. During the test, we performed standard maintenance such as filling and changing the plastic lubricant refill according to the instructions provided by the vehicle manufacturer. During the trial period $(125000 \mathrm{~km}, 3300$ OpHrs), we took 42 samples of plastic lubricant at relatively short intervals and analysed them. The samples were analysed with an AES Spectroil Q100 atomic emission spectrometer. The concentration of $\mathrm{Fe}$ abrasion metal in the plastic lubricant was selected for the analysis using the atomic emission spectrometry method AES/RDE. When a certain content of metals and particles was observed during the test of the bearing box, an increase in wear was detected during the period covering $20000 \mathrm{~km}$. 


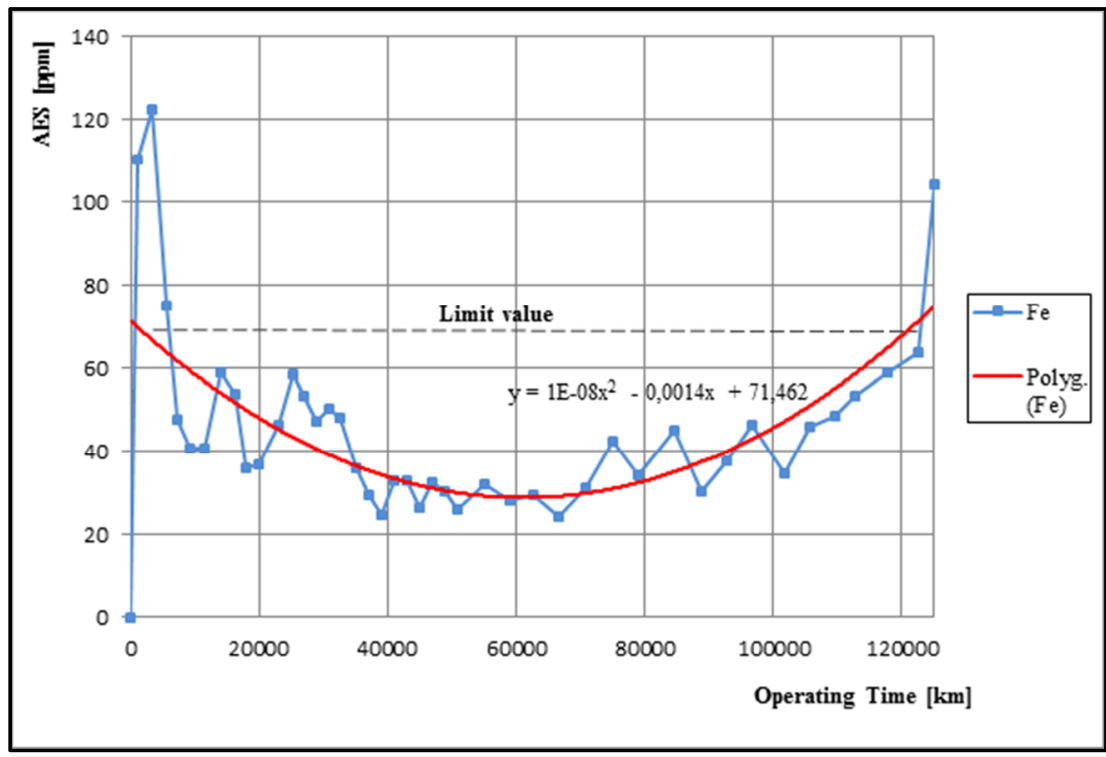

Fig. 15 Dependence of Fe in the lubricant on the operating time

We also noticed a significant increase in the Fe content due to a greater extent of wear caused by the failure in the frictional points of the bearings during the period covering more than $120000 \mathrm{~km}$ (see Fig. 15). For the modelling of an increase and a decrease in the $\mathrm{Fe}$ content, we used the polynomial course of trend with a generic formula (see Fig. 15).

\section{Conclusion}

The aim of the experiment was to verify whether the vibrodiagnostics is the right method of determining the technical condition of rotating elements. The attention was paid to roller bearings since they are among the most important parts of a machine transmission device.

Based on the performed measurements we can state that the vibration signal is a key signal when it comes to determining the technical condition of a rotating part of a vehicle. When processing the signal, it is necessary to take into account aspects which may create certain difficulties, e.g. determining a proper place for putting sensors, selecting right sensors, proper setting of software used for the evaluation, presenting the measurement results correctly, etc. The tools we selected for the measurement and the analysis were the Dewesoft company's software and hardware which we considered to be very suitable. Along with the DEWEsoftX2 software we also used the DIAdem software product which enabled us to put the results in graphs, see Fig. 9.

The experiment helped us to verify single stages and damage zones of the roller bearings shown in Fig. 5. In our experiment we explored in greater detail the vibration signal magnitude ranging from $3.5 \mathrm{kHz}$ up to $5 \mathrm{kHz}$ during the service life of a bearing in the bearing box. The results of the measured values are shown in Fig. 9. They include the measurements taken at the mileage of $0,30000,60000,90000$ and $122500 \mathrm{~km}$, which were later processed and presented in this figure. According to the theory developed in [5], the measured acceleration values should increase during the bearing service life. However, in our experiment the measured acceleration values decrease, which might have been caused by the facts that the roller bearing was not loaded and the bearing play was constantly increasing. During the measurements, the play was not intentionally adjusted.

In the next step we verified the theoretically calculated value of damage to the outer ring, see Tab. 1 and performed the experiment. The results which are shown in Fig. 12 were obtained by using the envelope detection. 
The experiment performed in our study shows that the $R M S$ value changes depending on the bearing mileage. We also found out that a higher RPM entails a higher RMS value. Therefore, it is rather complicated to determine the limiting value for replacing the bearing. Hence, it is necessary to determine different $R M S$ limiting values for different bearing revolutions.

\section{Acknowledgement}

The presented study has been prepared with the support of the Ministry of Defence of the Czech Republic, Partial Project for Institutional Development and Specific Research, Department of Combat and Special Vehicles, University of Defence, Brno.

\section{REFERENCES}

[1] Bartelmus, W. and Zimroz, R. A new feature for monitoring the condition of gearboxes in non-stationary operating conditions. Mechanical Systems and Signal Processing [online]. 2009, vol. 23, no. 5, p. 15281534. https://doi.org/10.1016/j.ymssp.2009.01.014

[2] Cong, F., Chen, J., Dong, G. and Pecht, M. Vibration model of rolling element bearings in a rotor-bearing system for fault diagnosis. Journal of Sound and Vibration [online]. 2013, vol. 332, no. 8, p. 2081-2097. https://doi.org/10.1016/j.jsv.2012.11.029

[3] Furch, J., Glos, J. and Blecha, J. Identifying the Technical Condition of Rotating Parts by Means of Vibrodiagnostics. Transaction of Famena 2015. vol. 39, no. 2, pp. $75-86$.

[4] Furch, J. Advanced maintenance systems of military vehicles. In: Proceedings of 9th International Conference ITELMS'2014. Panevezys: Kaunas University of Technology, 2014, p. 96-103.

[5] Girdhar, P. and Scheffer, C. Practical machinery vibration analysis and predictive maintenance. Burlington: Newnes, 2004, 255 p.

[6] Guo, Y., Eritenel, T., Ericson, T. M. and Parker, R. G. Vibro-acoustic propagation of gear dynamics in a gear-bearing-housing system. Journal of Sound and Vibration [online]. 2014, vol. 333, no. 22, p. 57625785. https://doi.org/10.1016/j.jsv.2014.05.055

[7] He, S., Singh, R. and Pavic, G. Effect of sliding friction on gear noise based on a refined vibro-acoustic formulation. Noise Control Engineering Journal [online]. 2008, vol. 56, no. 3, p. 164 - 175. https://doi.org/10.3397/1.2938737

[8] Kreidl, M. and Šmíd, R. Technical diagnosis: sensors, methods, signal analysis. Praha: BEN, 2006, 406p.

[9] Lim, T. Ch. and Singh, R. Statistical energy analysis of a gearbox with emphasis on the bearing path. Noise Control Engineering Journal [online]. 1991, vol. 37, no. 2, p. 63 - 69. https://doi.org/10.3397/1.2827795

[10] Product data: Tapered roller bearings 31308-A. Medias: Schaeffler [online]. [cit. 2015-05-19]. Available from: http://medias.schaeffler.de/medias/en!hp.ec.br.pr/ 313*31308-A

[11] Reeves, Ch. W. Vibration monitoring handbook. Oxford: Information Press, 1998.

[12] Vekteris V., Jurevicius M., Cereska A. Vibrodiagnostics of the components of technological machines. 8th International Conference on Vibration Measurements by Laser Techniques Location. Ancona (2008). https://doi.org/10.1117/12.803180

[13] Tsyfanskii S., Beresnevich V. Vibrodiagnostics of fatigue cracks in flexible, geometrically nonlinear, beam-type structural elements. Russian Journal of Nondestructive Testing (1994). pp 427-433

[14] Weber, Manfred. Triaxial-Beschleunigungsaufnehmer: KS943B100 [online]. 2014 [cit. 2015-05-19]. Available from: http://www.mmf.de/pdf/1-8.pdf

[15] Novotný, P., Prokop, A., Zubík, M. and Řehák, K. Investigating the influence of computational model complexity on noise and vibration modeling of powertrain. Journal of Vibroengineering. 2016, vol. 22 , no. 4, p. 277-392.

Submitted: $\quad 02.6 .2016$

Accepted: $\quad 01.02 .2017$
Prof. Eng. Jan Furch, Ph.D.

Eng. Josef Glos, Ph.D.

Eng. Trung Tin Nguyen, Ph.D.

Department of Combat and Special Vehicles

University of Defence

Kounicova str., 65 Brno, Czech Republic

E-mail: jan.furch@unob.cz 